\section{Soil Mechanics Laboratory}

Main activities: tests on soils (identification, static and dynamic), special tests (calibration of site equipment by means of calibration chambers, tests on geotechnical models also by means of centrifuge, thermomechanical tests), supply and installation of instruments, systems and software for static and dynamic testing of soils, research and development of original instrumentation and methodologies, data acquisition/processing systems, system design.

Instrumentation and System Dept.

Main activities: design, implementation and operation of measuring and monito- ring systems (setting up of measuring instruments, design and operation of hardware and software for structural surveillance), control and carrying out of tests (excitation devices, automation of soil testing equipment and mechanical loading systems), calibration and maintenance of electronic instrumentation, training of personnel on system operation.

Among the most recent testing facilities:

\section{Large shaking table}

Mode of operation: two axes and three rotations simultaneously, or any combination of those five degrees of freedom: dimensions: $4 \mathrm{~m} \times 4 \mathrm{~m}$;

weight: $110 \mathrm{kN}$;

maximum weight of the element to be tested: $300 \mathrm{kN}$;

frequency range: $0-120 \mathrm{~Hz}$;

peak to peak displacement: $200 \mathrm{~mm}$.

\section{Geotechnical centrifuge:}

dimensions: diameter $4 \mathrm{~m}$ rotating mass: $10,000 \mathrm{~kg}$;

mass of the model + container: $400 \mathrm{~kg}$; capacity: $\mathbf{2} 80 \mathrm{gt}$;

maximum acceleration: $700 \mathrm{~g}$

\title{
LABORATOIRE D'ESSAIS DES MATÉRIAUX
}

\section{Luxembourg}

\section{ATTRIBUTIONS}

Suivant la loi, le service du Laboratoire d'analyses et d'essais de matériaux est chargé :

- de l'étude et du contrôle des matériaux destinés aux services publics;

- des analyses chimiques et technologiques de ces matériaux, ainsi que

- des essais de contrôle sur leur mise en œuvre.

Ces prestations peuvent être faites également pour le compte d'entreprises privées et de particuliers.

\section{STRUCTURE}

Le Laboratoire est dirigé par un ingénieur-chimiste, le personnel est au nombre de 15 , de niveaux d'études différents.

\section{DOMAINES D'ACTIVITES}

Les divers domaines d'activité du Laboratoire sont les suivants :

- les liants et enrobés routiers, examinés régulièrement lors de leur mise en œuvre:

- le contrôle des postes d'enrobage;

- l'examen in situ et au Laboratoire des sols et fondations de routes;

- Ihomologation des produits de marquage routiers, en particulier des peintures blanches et des billes de verre réfléchissantes:

- l'examen du béton de ciment mis en cuvre sur les chantiers publics et privés:

- le contrôle régulier des centrales à béton:

- l'examen des granulats, des sables et des ciments:

- l'étude des pierres de construction, ainsi que l'examen des matériaux de construction en général.
Les principales activités de recherche sont :

- la mise au point des formulations de produits hydrocarbonés routiers nouveaux, exemple : (Schlammage):

- l'étude de bétons spéciaux, d'adjuvants, etc.

\section{EQUIPEMENT}

\section{(a) Essais des matériaux}

Une presse hydraulique pour essais de compression courant de cubes de béton (capacite $3000 \mathrm{kN}$ ).

Une presse électronique à indication digitale pour tous les essais mécaniques spéciaux tels que: traction, flexion, compression, arrachement, etc. (capacité $50 \mathrm{kN}$ ).

Une presse électronique pour les essais de compression des mortiers normalisés de ciment.

\section{(b) Essais des ciments}

Equipement permettant le contrôle suivant les normes DIN et NF.

\section{(c) Essais sur liants et enrobés hydro- carbonés}

Les liants hydrocarbonés : bitume, goudron-bitume, émulsion, sont examinés suivant les normes DIN ou belges.

Equipement courant.

Les enrobés hydrocarbonés sont analysés par extraction au solvant, en vue de la détermination de la teneur en liant, en filler et de la granulométrie.

Le laboratoire est également équipé pour préparer les éprouvettes et pour faire des essais mécaniques suivant Duriez et suivant Marshall (en conformité avec les normes allemandes et suisses). (d) Les granulats, sables, sols, fondations de routes

Equipement présent, permettant d'examiner ces matériaux au Laboratoire et in situ, en vue de déterminer leur granulométrie, leur teneur en matières fines, argiles, leur résistance à la compression statique. etc.

Les sols et fondations sont examines in situ dans le but de mesurer leur portance et leur degré de compactage. Ce dernier est mesuré par le biais de la densité in situ par gammadensimétrie, comparée à la densité optimale mesurée au Laboratoire suivant la méthode Proctor.

\section{(e) Les produits de marquage routier}

Les peintures routières sont homologuées régulièrement d'après des essais de laboratoire et in situ. L'équipement est simple, il permet de déterminer la viscosité, la densité, la composition, le pouvoir couvrant, d'adhérence du film, le temps de séchage, le vieillissement, etc.

\section{DIVERS TRAVAUX}

En dehors des grands travaux effectués dans l'intérèt des chantiers de routes et de travaux publics tels que ponts, barrages, etc., le laboratoire d'essais s'occupe également d'analyses chimiques diverses, ayant une relation avec le batiment, telles que : études de problèmes de corrosion, de mortiers de chape ot de façade, de plâtre, de revêtements pour planchers, de toitures, de pierres à construire artificielles et naturelles, etc. A cet effet, le laboratoire dispose de l'équipement classique pour analyses chimiques et d'un spectrophotomètre à infrarouge pour analyses spéciales. 